\title{
1.1. Foreword
}

This volume stems from University of Zagreb Round Table 2016 (UZRT 2016) conference, a yearly event which is organized alternately by the University of Pecs and the University of Zagreb. The event started as a platform through which younger researchers would be given the opportunity to receive feedback on their research from peers and more experienced colleagues.

The volume comprises 12 papers which cover various empirically studied topics and contribute to the wealth of knowledge in the field of applied linguistics. Some studies propose new theoretical models, and other have a narrower focus and inquire into specific issues of concern to the field of language learning and use. We have broadly categorized them into two areas of interest; studies pertaining to the assessment of skills, prospects and outcomes, and those investigating individual differences, diversity and learners with disabilities.

The first seven papers in this volume address the topic of assessment of either competencies and skills acquired through different educational courses and programs, or pedagogical prospects and value of instructional material and assessment tools. Out of the remaining five papers in the second part of the volume, three papers deal with individual differences, or more specifically, affective aspects in the process of language acquisition and use, and two papers deal with the characteristics and needs of learners with disabilities and difficulties.

Even though most of the papers have the English language as their focus, we believe that the range of educational and sociolinguistic settings as well as different age groups of the participants included in the analyses make the volume appealing to a wider audience of researchers, teachers and students, as well as curriculum designers and developers.

We would like to thank the authors and reviewers for their thoughtful contributions as well as professors Marianne Nikolov and Jelena Mihaljević Djigunović, the initiators of this valuable collaboration between the two universities, for their support to this yearly event.

The editors 